\title{
6. Umfrage «Weiterbildungsqualität - Bewertung durch die Assistentinnen und Assistenten»
}

Es ist wieder soweit: nach der erfreulichen Rücklaufquote von 74\% im Jahr 2001 (SÄZ 2002;83 [24]:1227-8) wird die Umfrage «Weiterbildungsqualität - Bewertung durch die Assistentinnen und Assistenten» dieses Jahr bereits zum sechsten Mal durchgeführt. Alle Ärztinnen und Ärzte in Weiterbildung sind dazu aufgerufen, den gelben Fragebogen auszufüllen. Dadurch tragen sie einen wichtigen Teil zur Qualitätssicherung der ärztlichen Weiterbildung bei.

Die Umfrage erfolgt zum letzten Mal mit diesem Fragebogen. Der Zentralvorstand hat im Juli 2002 den Kredit gesprochen für die Schaffung eines neuen Fragebogens, der den Anregungen und Kritiken Rechnung tragen soll. Im letzten Quartal 2002 soll in Arbeitsgruppen mit Leiterinnen und Leitern der Weiterbildungsstätten und Assistentinnen und Assistenten ein neuer Fragebogen erarbeitet werden, der im Spätsommer 2003 zum Einsatz kommt. Die Fragen sollen detaillierter den Weiterbildungsprozess bezüglich Inhalt und Qualität beleuchten, so dass aufgrund deren Beantwortung konkrete Massnahmen eingeleitet werden können.
Mitte August werden die Leiterinnen und Leiter der Weiterbildungsstätten von der FMH mit den gelben Fragebogen bedient und gebeten, die Fragebogen an die Weiterzubildenden zu verteilen und durch deren Vertreterin oder Vertreter einsammeln und an die FMH zur Auswertung weiterleiten zu lassen (die Fragebogen können selbstverständlich auch von jedem/jeder einzelnen Assistenten/-in direkt an das Sekretariat Aus-, Weiter- und Fortbildung [AWF] der FMH retourniert werden).

Die Anzahl der Ärztinnen und Ärzte in Weiterbildung und damit die Anzahl der zuzustellenden Fragebogen wurde bei den Leiterinnen und Leitern der Weiterbildungsstätten in einer vorausgegangenen statistischen Erhebung ermittelt. Nur Ärztinnen und Ärzte in Weiterbildung erhalten einen gelben Fragebogen!

Wir zählen weiterhin auf eine hohe Teilnahme der Weiterzubildenden an der Umfrage und danken ihnen für den Einsatz im Interesse der ärztlichen Weiterbildung. 